\title{
The World at 7:00: Comparing the Experience of Situations Across 20 Countries
}

\author{
Esther Guillaume, Erica Baranski, Elysia Todd, \\ Brock Bastian, Igor Bronin, Christina Ivanova, \\ Joey T. Cheng, François S. de Kock, Jaap J. A. Denissen, \\ David Gallardo-Pujol, Peter Halama, Gyuseog Q. Han, \\ Jaechang Bae, Jungsoon Moon, Ryan Y. Hong, \\ Martina Hřebíčková, Sylvie Graf, Paweł Izdebski, \\ Lars Lundmann, Lars Penke, Marco Perugini, \\ Giulio Costantini, John Rauthmann, Matthias Ziegler, \\ Anu Realo, Liisalotte Elme, Tatsuya Sato, \\ Shizuka Kawamoto, Piotr Szarota, Jessica L. Tracy, \\ Marcel A. G. van Aken, Yu Yang, and David C. Funder
}

\begin{abstract}
The purpose of this research is to quantitatively compare everyday situational experience around the world.

Local collaborators recruited 5,447 members of college communities in 20 countries, who provided data via a Web site in 14 languages. Using the 89 items of the Riverside Situational Q-sort (RSQ), participants described the situation they experienced the previous evening at 7:00 p.m.

Correlations among the average situational profiles of each country ranged from $r=.73$ to $r=.95$; the typical situation was described as largely pleasant. Most similar were the United States/Canada; least similar were South Korea/Denmark. Japan had the most homogenous situational experience; South Korea, the least. The I5 RSQ items varying the most across countries described relatively negative aspects of situational experience; the 15 least varying items were more positive. Further analyses correlated RSQ items with national scores on six value dimensions, the Big Five traits, economic output, and population. Individualism, Neuroticism, Openness, and Gross Domestic Product yielded more significant correlations than expected by chance.

Psychological research traditionally has paid more attention to the assessment of persons than of situations, a discrepancy that extends to cross-cultural psychology. The present study demonstrates how cultures vary in situational experience in psychologically meaningful ways.
\end{abstract}

People around the world are in some respects different and in some respects the same (Kluckhohn \& Murray, 1953, p. 53) and so, too, are the situations they experience (Brown, 1991). However, despite the widespread acknowledgement that behavior is a function of both the person and the situation, researchers have traditionally paid more attention to the assessment of attributes of the former than the latter (Bem \& Funder, 1978; Funder, 2009; Sherman, Nave \& Funder, 2010; Wagerman \& Funder, 2009), and this difference extends to cross-cultural psychology.

Numerous investigations have identified differences in the ways people differ psychologically across countries (Church, $2010)^{1}$. These differences include social orientation, e.g., interdependent vs. independent styles of self-construal (Markus \& Kitayama, 1998 ; Masuda \& Nisbett, 2001); personality struc- ture, e.g., the degree to which the "Big Five" traits account for individual differences in diverse cultures (Gurven, von Rueden, Massenkoff, \& Kaplan, 2013; McCrae \& Allik, 2002; McCrae \& Costa, 1997); and the degree to which international differences in personality match national stereotypes (Hřebíčková \&

Author affiliations are listed in the Appendix.

Data gathering was assisted by Joanne Fullerton, Danique van den Hanenberg, Sakiko Kumagai, and Elizabet Orekhova. Portions of the U.S. and Japanese data were utilized in an earlier paper (Funder, Guillaume, Kumagi, Kawamoto, \& Sato, 2012), but all of the analyses reported here are new.

Correspondence concerning this article should be addressed to David Funder, Department of Psychology, University of California, Riverside, CA 9252I USA. Email: funder@ucr.edu. 
Graf, 2014; McCrae et al., 2013; Realo et al., 2009; Terracciano et al., 2005). Recent studies have also addressed national differences in within-person behavioral variability (Ching et al., 2013; Church et al., 2013).

Research has paid less attention to the experience of everyday situations. The traditional method for assessing the cultural environment has been anthropological fieldwork, in which skilled researchers immerse themselves in unfamiliar settings, interview informants, and return with detailed or "thick" descriptions (Shweder, 1991). However, this style of anthropology generally eschews cross-cultural comparison, which is sometimes seen as inimical to full understanding (Frake, 1980).

Within psychology, Gelfand et al. (2011) asked participants in 33 countries to rate the degree to which social norms constrained behavior in briefly described hypothetical settings (e.g., bank, park, bedroom), and Church, Katigbak, and del Prado (2010) gathered ratings of the degree to which hypothetical situations were conducive to expressing the Big Five personality traits. Other pioneering studies asked participants in the United States and Japan to recall situations relevant to their self-esteem (Kitayama, Markus, Matsumonot, \& Narasakkunkit, 1997) and emotions (Kitayama, Mesquita, \& Miyamoto, 2006). Such research remains rare, and none of these groundbreaking efforts comprehensively compared ordinary daily situations assessed from the viewpoint of persons who had recently experienced them. However, this type of assessment is important, because the environments people encounter every day manifest both universal and specific aspects of culture. Indeed, to the extent that people differ across cultures, this is almost certainly due to their experiences while living in them (Brown, 1991; Oyserman, in press; Triandis, 1996).

The paucity of cross-cultural research on situational experience may stem from the lack of a comprehensive assessment tool. Creative researchers have addressed the classification of situations within single countries (Bem \& Funder, 1978; Edwards \& Templeton, 2005; Forgas \& van Heck, 1992; Kelley et al., 2003; Magnusson, 1971; Reis, 2008; ten Berge \& De Raad, 1999; Van Heck, 1984; Yang, Read \& Miller, 2006). However, none of their work yielded a usable measurement instrument, much less one that could be applied cross-culturally. The Riverside Situational Q-sort (RSQ) is a step toward filling this gap.

\section{THE RIVERSIDE SITUATIONAL Q-SORT (RSQ)}

The recognition that behavior is a function of the interaction between the person and the situation implies a need for comparable measurement of all three elements of the "personality triad" (Funder, 2006, 2009). The 100-item California Adult Q-sort (CAQ) has long been available as a comprehensive measure of personality (Block, 1978). The 68-item Riverside Behavioral Qsort (RBQ) was developed several years ago as a parallel means to assess behavior (Funder, Furr, \& Colvin, 2000; Furr, Wager- man \& Funder 2010). The RSQ was developed more recently as the third member of this group of instruments (Wagerman \& Funder, 2009).

Various writers have agreed that situations can be conceptualized at three basic levels (Block \& Block, 1981; Gelfand, 2007; Saucier, Bel-Behar \& Fernandez, 2007; Wagerman \& Funder, 2009). The first level is Macro/Physico-biological/Environmen$\mathrm{tal}$, which includes climate, location, and number of people in the room; ecological, historical, and sociopolitical factors; and possibly physiological arousal, but does not include psychological aspects of the situation as separate from these specific, objective properties. The second level, Meso/Canonical/Consensual, refers to psychological aspects of the situation that, while intangible, can be widely agreed upon by competent social observers. The final level, Micro/Subjective/Functional, defines properties of situations in terms of individuals' (perhaps idiosyncratic) perceptions of them. The RSQ aims at the Meso/Canonical/Consensual level of analysis, with 89 items including "Situation is potentially emotionally arousing," "Others are present who need or desire reassurance," and "Situation is potentially enjoyable."

Participants use the RSQ to describe their situational experience by arranging its 89 items into a 9-step, forced-choice distribution ranging from "highly uncharacteristic" (category 1), to "highly characteristic" (category 9). The distribution is quasinormal, with the bulk of the items directed to the middle categories. The few items that make it into the extreme, 1 or 9 categories therefore scale a high hurdle of being judged more descriptive than the vast majority of the others.

\section{UTILIZING THE RSQ FOR CROSS- CULTURAL RESEARCH}

Appropriate measurement is critical in cross-cultural research. In particular, research should (a) avoid imposing complex constructs that may not replicate across cultures, (b) carefully backtranslate items that are as free as possible of cultural idioms, and (c) administer measurements in a uniform manner across cultures (see van de Vijver and Leung, 2011, for a review). The present study using the RSQ takes all of these issues into consideration.

First, the RSQ items were not determined or restricted by any particular theory. They were largely based on the comprehensive set of personality-descriptive items provided by the CAQ. For example, the first item of the CAQ reads, "Is critical, skeptical, not easily impressed." The first item of the RSQ is "Someone is trying to convince $\mathrm{P}$ (the person in the situation) of something." The presumption is that a skeptical person would react to this situation one way; a credulous person in the opposite way. (See Wagerman \& Funder, 2009, for more details on the development of the RSQ.) For the purposes of the present study, we developed a new version of the RSQ to minimize the use of jargon and make translations easier (Funder \& Guillaume, 2013).

Next, the forced-choice format helps reduce the influence of response styles that can plague cross-cultural research (Ross \& Mirowsky, 1984; van de Vijver \& Leung, 1997, 2011). For 
example, "acquiescence bias," the tendency to agree with items regardless of their content, is eliminated by the RSQ because a set number of items are placed into each evaluative category. The "social desirability" bias, the tendency to rate desirable items high and undesirable items low, is reduced (if not wholly eliminated) because the highest and lowest rating categories are not large enough to contain all of the desirable and undesirable items, respectively. Additionally, the halo effect, which is the tendency to rate groups of semantically related items similarly to each other, is attenuated because it is simply not possible to put all items of a type (e.g., all socially desirable items) into a single category.

Finally, forced-choice measures also help address reference group effects, in which participants make ratings in comparison to other members of their own peer (or cultural) group, a tendency which could deflate cross-cultural differences (Heine, Lehman, Peng, \& Greenholtz, 2002). With forced-choice measures, raters compare items to each other rather than to normative expectations. For example, a rater completing the RSQ must decide whether "Someone is trying to convince someone of something" is a more salient descriptor of a particular situation than "A job needs to be done," but does not have to rate whether either of these items is higher or lower than it would be when describing other situations within his or her culture. Perhaps surprisingly, given these advantages, forced-choice measures such as the Q-sort are infrequently employed in cross-cultural research, ${ }^{2}$ despite suggestions that they might be helpful (Heine et al., 2002; McCrae et al., 2013).

\section{RESEARCH QUESTIONS ADDRESSED BY Q-SORT ANALYSES}

The ability of the RSQ to comprehensively measure situational similarity opens new research questions. One of the first published studies on this topic found that situations experienced over time by a given participant tend to be described more similarly to each other than to situations experienced by others (Sherman et al., 2010). Moreover, behavior is more consistent across situations described more similarly, and personality characteristics predict individual degrees of behavioral consistency, even after statistically controlling for situational consistency. Further studies found that the degree to which one's personality matches or is "congruent" with behavior in particular situations is associated with psychological adjustment (Sherman, Nave \& Funder, 2012), and that construing a situation "distinctively" (i.e., differently from most other observers) is associated with personality attributes including Neuroticism and Openness (Serfass \& Sherman, 2013; Todd \& Funder, 2012). The RSQ has also been used to construct prototypical templates of situational categories suggested by evolutionary theory, allowing behavioral predictions to be empirically tested (Morse, Neel, Todd, \& Funder, 2014).

Examination of individual RSQ items can also be informative. For example, extraverts are more likely to see themselves as the focus of attention, and men are more likely than women to see a potential for someone to be blamed for something (Sherman, Nave, \& Funder, 2013). And, the first cross-cultural application of the RSQ found that behavioral correlates of items including "P [the participant rating his or her own situation] is being criticized" and "Members of the opposite sex are present" were remarkably similar in the United States and in Japan (Funder, Guillaume, Kumagi, Kawamoto, \& Sato, 2012). This last finding is an encouraging indication that the RSQ may be suitable for cross-cultural research.

\section{THE PRESENT STUDY}

The present study is frankly exploratory, seeking to provide a base for future inductive theory construction (Haig, 2005, 2014). More specifically, the present research has four goals:

1. Assess the experience of situations by people around the world on an ordinary day at 7:00 p.m., as reported less than 24 hours later. The time of 7:00 p.m. was chosen because a wide variety of possible activities may occur at that hour, ranging from eating dinner to socializing to working. Reporting on a situation experienced the previous evening may also help to minimize memory distortion or selective reporting.

2. Examine the degree to which situational experience at this hour is similar and different. Questions include: Which countries, on average, have the most and least similar situational experience at 7:00 p.m.? Which countries have the most homogenous situational experience (experiences similar across participants within the country), and which countries are the most heterogeneous? How does the diversity of situations within countries compare to differences in situations between countries?

3. Examine specific differences in situational experience. Questions include: Which attributes of situations vary the most across countries? On those attributes, which countries have the highest and lowest means? What is the difference between the attributes that vary the most and least?

4. Correlate attributes of situational experience with attributes of countries including scores on values, average levels of the Big Five personality traits, economic data (gross national product), and population.

\section{METHOD \\ Participants}

All participants were members of college communities, primarily students, recruited by research collaborators in each of 20 countries on five continents, using 14 languages, with a total $\mathrm{N}=5,447 \quad$ (female $=3,488$, male $=1959 ;$ mean age $=22$ years). Table 1 provides details. From an original sample of 5,485 participants, 22 were deleted for apparently reversing the 
Table I Samples from 20 Countries

\begin{tabular}{|c|c|c|c|c|c|c|}
\hline Country & Language of Assessment ${ }^{a}$ & Compensation & $\mathrm{N}$ & Female & Male & Mean Age \\
\hline Australia & English & Course credit & $|4|$ & 109 & 32 & 20 \\
\hline Austria & German & Volunteer & 87 & 71 & 16 & 25 \\
\hline Canada & English & Course credit & 191 & 126 & 65 & 21 \\
\hline China & Simplified Chinese & $\$ 0.67$ per person & $\mathrm{I}, 565$ & 854 & 711 & 22 \\
\hline Czech Republic & Czech & Volunteer & 220 & 159 & 61 & 28 \\
\hline Denmark & Danish & Volunteer & 126 & 102 & 24 & 23 \\
\hline Estonia & Estonian & Volunteer & 314 & 251 & 63 & 26 \\
\hline Germany & German & Course credit & 70 & 55 & 15 & 27 \\
\hline Italy & Italian & Course credit & 144 & 75 & 69 & 23 \\
\hline Japan & Japanese & Volunteer & 227 & 107 & 120 & 20 \\
\hline Netherlands & Dutch & Course credit & 258 & 220 & 38 & 20 \\
\hline Poland & Polish & Volunteer & 97 & 73 & 24 & 24 \\
\hline Russia & Russian & Course credit & 101 & 80 & 21 & 21 \\
\hline Singapore & English & Course credit & 158 & 109 & 49 & 21 \\
\hline Slovakia & Slovak & Volunteer & 98 & 86 & 12 & 22 \\
\hline South Africa & English & Course credit & 114 & 62 & 52 & 23 \\
\hline South Korea & Korean & Course credit & 103 & 69 & 34 & 22 \\
\hline Spain & Spanish & Volunteer & 108 & 78 & 30 & 22 \\
\hline UK & English & Course credit & 107 & 75 & 32 & 21 \\
\hline United States & English & Course credit & 1,218 & 727 & 491 & 20 \\
\hline
\end{tabular}

Total $\mathrm{N}=5,447$ : females: 3,488 , males: 1,959 .

${ }^{a} \mathrm{~A}$ small number of participants elected to respond in a language other than the country's primary language. One participant (each) in Australia, Canada, Estonia, and the United States responded in Chinese. Two participants in the Netherlands responded in German. Five participants in the Czech Republic responded in Slovak. One participant in Austria responded in English, as did two in Estonia and twenty-four in China

response scale and 16 were deleted because of other failures to follow instructions or due to incomplete data.

\section{Data-Gathering Web Site}

Data were gathered via a custom-built Web site, which was required for three reasons: (1) available online platforms (e,g., Qualtrics; M-turk) could not support forced-choice measures or offer the drop-and-drag function necessary for completing Qsorts; (2) the Web site needed to support multiple languages, including those using non-Roman characters; and (3) several research locations lacked facilities for participants to complete measures in a lab. An interested reader can repeat the experience of a participant, in English, by going to this Web site (www. internationalsituationsproject.com), clicking on the U.S. flag icon, and entering "amtest1" as the Study ID and "am001" as the participant ID (data entered will not be saved).

\section{Procedure}

Collaborators distributed login IDs to participants within each of the collaborators' countries. The Web site asked participants to provide basic demographic information, and then to briefly describe, in their own words, the situation they experienced the previous evening at 7:00 p.m. Participants were instructed to describe only one situation, reporting (1) where they were, (2) who they were with, and (3) what they were doing. If a participant was sleeping, he or she was asked to describe what happened right before going to sleep, or right after waking.
Examples of situational descriptions included: "My cousin came over and we were relaxing on the balcony after a day of snowboarding. We were smoking cigarettes and drinking wine" (United States); "I was at my grandma's house eating dinner. I was with my cousins, aunts, uncles, grandma and my own family" (Singapore); "I sang using the karaoke box with my friend" (Japan); "I was cooking pizza with my boyfriend" (Italy); "At about 7 I was sitting in the sauna with my grandmother, adding some steam and whisking" (Estonia). Finally, participants described the situation using the Riverside Situational Q-Sort (RSQ).

\section{Assessment of Situational Experience}

The Riverside Situational Q-sort 3.15 (RSQ; Funder and Guillaume, 2013; previous version 2.0, Wagerman \& Funder, 2009). Table 2 displays the 89 items along with their overall mean placement across 20 countries. These items, as well as the online instructions to participants, were translated from English into 13 other languages by our local research collaborators, and then independently back-translated. The original and translated versions were then compared, and discrepancies resolved before the final translation was settled. For example, the initial translation into Japanese of item 77, "Affords an opportunity to express charm," was back-translated as "Affords an opportunity to express attraction." The initial translation into Estonian of item 35 , "A person or activity could be undermined or sabotaged," was back-translated as "A person or act could be hallowed or sabotaged." Such discrepancies were actually rather rare, and all 
Table 2 Riverside Situational Q-sort Version 3.I5

\begin{tabular}{|c|c|c|}
\hline RSQ \# & RSQ Situational Item & Overall Mean \\
\hline rsq076 & Situation is basically simple and clear-cut. & 7.01 \\
\hline rsq056 & Social interaction is possible. & 6.64 \\
\hline rsq00I & Situation is potentially enjoyable. & 6.42 \\
\hline rsq007 & Talking is permitted. & 6.31 \\
\hline rsq046 & Situation allows a free range of emotional expression. & 6.00 \\
\hline rsq059 & Situation includes sensuous stimuli (e.g., touch, taste, smell, physical contact). & 5.85 \\
\hline rsq05I & Close personal relationships are present or have the potential to develop. & 5.83 \\
\hline rsq025 & Rational thinking is called for. & 5.81 \\
\hline rsq083 & Situation is potentially emotionally arousing. & 5.81 \\
\hline rsq003 & A job needs to be done. & 5.79 \\
\hline rsq073 & Members of the opposite sex are present. & 5.79 \\
\hline rsq063 & Others present a wide range of interpersonal cues (e.g., body language, tone of voice, social signals). & 5.78 \\
\hline rsq049 & Affords an opportunity to ruminate, daydream, or fantasize. & 5.76 \\
\hline rsq008 & Talking is expected or demanded. & 5.72 \\
\hline rsq018 & Situation is playful. & 5.72 \\
\hline rsq067 & Situation includes explicit or implicit demands on P. & 5.70 \\
\hline rsq0II & Minor details are important. & 5.66 \\
\hline rsq057 & Situation is humorous or potentially humorous (if one finds that sort of thing funny). & 5.65 \\
\hline rsq006 & $P$ is counted on to do something. & 5.64 \\
\hline rsq0I3 & $\begin{array}{l}\text { Affords an opportunity to demonstrate intellectual capacity (e.g., an intellectual discussion, a complex } \\
\text { problem needs to be solved). }\end{array}$ & 5.64 \\
\hline rsq024 & A decision needs to be made. & 5.63 \\
\hline rsq053 & Situation includes intellectual or cognitive stimuli (e.g., books, lectures, intellectual conversation). & 5.63 \\
\hline rsq035 & Situation might evoke warmth or compassion. & 5.63 \\
\hline rsq028 & Affords an opportunity for $\mathrm{P}$ to do things that might make $\mathrm{P}$ liked or accepted. & 5.62 \\
\hline rsq084 & $\begin{array}{l}\text { Affords an opportunity for demonstrating verbal fluency (e.g., a debate, a monologue, an active } \\
\text { conversation). }\end{array}$ & 5.62 \\
\hline rsq019 & $\begin{array}{l}\text { Introspection is possible (e.g., the atmosphere allows or encourages reflection upon deeply personal } \\
\text { issues). }\end{array}$ & 5.54 \\
\hline rsq009 & $P$ is being asked for something. & 5.54 \\
\hline rsq04I & Affords an opportunity to express unusual ideas or points of view. & 5.53 \\
\hline rsq064 & Situation includes behavioral limits. & 5.50 \\
\hline rsq087 & Success requires cooperation. & 5.50 \\
\hline rsq034 & Situation includes one or more small annoyances. & 5.46 \\
\hline rsq065 & Situation includes aesthetic stimuli (e.g., art, music, drama, beauty). & 5.45 \\
\hline rsq068 & Affords an opportunity to express or demonstrate ambition. & 5.44 \\
\hline rsq032 & It is important for $\mathrm{P}$ to make a good impression. & 5.40 \\
\hline rsq026 & Situation calls for self-restraint. & 5.37 \\
\hline rsq055 & $\begin{array}{l}\text { Situation includes potential for immediate gratification of desires (e.g., food, shopping, sexual } \\
\text { opportunities). }\end{array}$ & 5.36 \\
\hline rsq085 & People who are present occupy different social roles or levels of status. & 5.34 \\
\hline rsq052 & Someone (other than P) is counted on to do something. & 5.29 \\
\hline rsq022 & A reassuring other person is present. & 5.28 \\
\hline rsq012 & Situation evokes values concerning lifestyles or politics. & 5.27 \\
\hline rsq077 & Affords an opportunity to express one's charm. & 5.20 \\
\hline rsq020 & Things are happening quickly (low placement implies things are happening slowly). & 5.18 \\
\hline rsq08I & Others may need or are requesting advice from $\mathrm{P}$. & 5.16 \\
\hline rsq06I & Success in this situation requires self-insight. & 5.14 \\
\hline rsq0I0 & Someone needs help. & 5.14 \\
\hline rsq078 & Situation involves social comparison. & 5.11 \\
\hline rsq005 & Someone is trying to convince $\mathrm{P}$ of something. & 5.10 \\
\hline rsq054 & Assertiveness is required to accomplish a goal. & 5.09 \\
\hline rsq033 & Situation would make some people tense and upset. & 5.01 \\
\hline rsq004 & Someone is trying to impress $\mathrm{P}$. & 4.97 \\
\hline rsq088 & $\mathrm{P}$ is being complimented or praised. & 4.94 \\
\hline rsq045 & A quick decision or quick action is called for. & 4.90 \\
\hline rsq030 & Situation entails frustration (e.g., a goal is blocked). & 4.90 \\
\hline rsq029 & Others are present who need or desire reassurance. & 4.89 \\
\hline
\end{tabular}


Table 2 (Continued)

\begin{tabular}{|c|c|c|}
\hline RSQ \# & RSQ Situational Item & Overall Mean \\
\hline rsq040 & People are disagreeing about something. & 4.87 \\
\hline rsq075 & Situation has potential to arouse competing motivations. & 4.86 \\
\hline rsq002 & Situation is complex. & 4.86 \\
\hline rsq058 & $P$ is the focus of attention. & 4.85 \\
\hline rsq027 & Situation involves competition. & 4.83 \\
\hline rsq0I4 & Situation is uncertain. & 4.77 \\
\hline rsq02I & Someone (present or discussed) is unhappy or suffering. & 4.54 \\
\hline rsq089 & Affords an opportunity to express femininity. & 4.48 \\
\hline rsq016 & $P$ is being criticized, directly or indirectly. & 4.47 \\
\hline rsq044 & Situation raises moral or ethical issues (e.g., a moral dilemma is present; a discussion of morality). & 4.46 \\
\hline rsq066 & Situation is potentially anxiety-inducing. & 4.43 \\
\hline rsq031 & Physical attractiveness (of $\mathrm{P}$ ) is relevant. & 4.38 \\
\hline rsq062 & $\mathrm{P}$ controls resources needed by others. & 4.36 \\
\hline rsq048 & Situation entails or could entail stress or trauma. & 4.33 \\
\hline rsq07। & Situational demands are rapidly shifting. & 4.32 \\
\hline rsq069 & Situation might make $\mathrm{P}$ feel inadequate. & 4.31 \\
\hline rsq047 & Others present might have conflicting or hidden motives. & 4.31 \\
\hline rsq017 & Someone is attempting to dominate or "boss" P. & 4.30 \\
\hline rsq036 & A person or activity could be undermined or sabotaged. & 4.29 \\
\hline rsq037 & It is possible for $\mathrm{P}$ to deceive someone. & 4.16 \\
\hline rsq039 & Situation may cause feelings of hostility. & 4.16 \\
\hline rsq050 & Situation has potential to arouse guilt (in $\mathrm{P}$ ). & 4.11 \\
\hline rsq0I5 & Another person (present or discussed) is under threat. & 4.09 \\
\hline rsq080 & Affords an opportunity to express masculinity & 4.08 \\
\hline rsq038 & Someone else in this situation (other than P) might be deceitful. & 4.06 \\
\hline rsq060 & Situation is relevant to P's bodily health (e.g., possibility of illness; a medical visit). & 4.06 \\
\hline rsq043 & Situation contains emotional threats. & 3.96 \\
\hline rsq086 & $\mathrm{P}$ is being pressured to conform to the actions of others. & 3.96 \\
\hline rsq079 & Situation raises issues of power (for $\mathrm{P}$ or others present). & 3.92 \\
\hline rsq023 & $\mathrm{P}$ is being blamed for something. & 3.89 \\
\hline rsq074 & Potential romantic partners (for $\mathrm{P}$ ) are present. & 3.86 \\
\hline rsq070 & Situation includes stimuli that could be construed sexually. & 3.38 \\
\hline rsq042 & Situation contains physical threats. & 3.31 \\
\hline rsq082 & P's independence and autonomy is questioned or threatened. & 3.17 \\
\hline rsq072 & $P$ is being abused or victimized. & 2.17 \\
\hline
\end{tabular}

Note. P refers to the person whose presence in the situation is at issue. Sorted in order of overall mean rating across 20 countries. Ratings are from a Q-sort distribution ranging from I (highly uncharacteristic) to 9 (highly characteristic).

were addressed in collaboration with our international collaborators before data gathering began.

Using a drag-and-drop function, participants began by sorting the 89 items of the RSQ, without restriction, into three categories: "uncharacteristic," "neutral," or "characteristic." This first step simplifies the final task of arranging the items into nine categories with a forced-choice distribution ranging from "extremely characteristic" to "extremely uncharacteristic." The numbers of items prescribed for each of the nine categories are as follows: $3,6,11,15,19,15,11,6$, and 3 .

In data analyses, the 89 item ratings from each participant can be compared as a "profile" with the 89 ratings provided any other participant, by computing conventional correlation coefficients. Each item rating can also be averaged across individuals within a country, yielding a single average profile that can be compared with the average profile of other countries. In addition, mean placements of individual items can be com- pared across countries, and correlated with country-level variables.

\section{RESULTS \\ Cross-Cultural Similarity}

For each country, we separated the samples by sex, and then averaged all participants' RSQ-sorts. This yielded one RSQsort for each sex and each country. We then averaged the male and female RSQ-sorts within each country. This way, both genders contributed equally to the composite. This procedure yielded a single list of 89 average RSQ-item placements for each country. These average Q-sorts can then be compared with each other using a standard Pearson correlation, yielding a $20 \times 20$ correlation matrix. The results appear in Table 3. 
These correlations are in general very high, with an average cross-cultural similarity of $r=.84$. The highest similarity in average situational experience, perhaps not surprisingly, was between the United States and Canada ( $r=.95 ; 95 \%$ CI [.93, 97], $\left.d f=87^{3}\right)$. The lowest similarity was between South Korea and Denmark ( $r=.73 ; 95 \%$ CI $[.61, .82], d f=87)$. The overall high degree of cross-cultural similarity draws our attention back to Table 2, which reports the overall mean placement of each RSQ item across 20 countries. The highest rated items, overall, included descriptions of situations as simple and clear-cut, social, and potentially enjoyable. The lowest rated items referred to experiences of abuse and victimization, threats to independence, and physical threats. Around the world, the typical situation at 7:00 p.m. can be described as a largely pleasant social interaction.

The bottom rows of Table 3 show the average similarity of each country to the other 19 , along with the confidence intervals around each mean. A conventional omnibus of variance demonstrates a significant difference among these means overall ( $F$ $[19,360]=9.71, p<.0001)$.

Canada was the country most similar to all the others (average $r=.89 ; 95 \%$ CI $[.88, .90], d f=19^{4}$ ); the most distinctive countries were South Korea and Japan (both, average $r=.80 ; 95 \%$ CI $[.79, .81], d f=19)$. In most cases, countries with more than .03 differences in their average correlations were outside each other's confidence intervals. Thus, even though all the correlations are rather high, the variation among them is meaningful. For example, Canada and the United States are almost identical in their average similarity to other countries, and are both less distinctive than Australia, Austria, Denmark, Japan, South Africa, and South Korea.

\section{Within-Country Homogeneity}

The correlations described so far are all between averages computed within each country. With these data, it is also possible to assess the degree of similarity of RSQ reports among individuals within each country. This analysis entails correlating the complete RSQ report of each individual with that of every other individual within the country and then averaging these correlations. We did this separately within each gender, and then averaged the two within-country correlations, which are bold-faced on the diagonal in Table 4. Not surprisingly, these numbers are much smaller than the correlations in Table 3, because they represent correlations among individuals rather than country wide mean profiles.

The correlations along the diagonal in Table 4 can be interpreted as measures of within-country homogeneity of situational experience-the degree to which situational reports by individuals tend to resemble those of other individuals (of the same gender) in the same country. The country with the most homogenous cultural experience at 7:00 p.m. Was Japan (within-country average $r=.28 ; 95 \%$ CI $[.26, .30], d f=225)$, and the least homogenous cultural expe- rience was within South Korea (average $r=.12 ; 95 \%$ CI $[.09, .15], d f=101)$.

Further analyses correlated the RSQ offered by each participant in each country with every other participant (of the same sex) in each of the other countries, and the average of these correlations is reported in the off-diagonal cells of Table 4. It is readily apparent that these between-country comparisons, while indeed smaller than the within-country comparisons, are not dramatically so, with the limits of the confidence intervals barely apart (within country average $r=.20,95 \%$ CI $[.183, .216]$, $d f=19$; across country average $r=.1895 \%$ CI $[.177, .182]$, $d f=189) .^{5}$ This finding underlines the conclusion drawn from the analyses in Table 3, which is that cross-cultural similarities seem to be a more notable feature of situational experience than cross-cultural differences.

\section{Cross-Cultural Differences in Attributes of Situational Experience}

The next step in the data analysis was to explore the specific attributes of situational experience that vary more and less across countries. Notwithstanding the overall high degree of similarity in experience noted in the previous section, there were many such differences. In fact, when an analysis of variance is performed on the differences in average placement of each of the RSQ items across countries, all 89 of the F's are significant at the conventional $p<.05$ level. However, this result is perhaps to be expected given that the overall $\mathrm{N}$ for each analysis is 5,447 .

\section{Positivity/Negativity and Cross-Cultural Variation}

More informative, therefore, is assessment of the effect size, which in this case is indicated by the eta $(\eta)$. The 15 RSQ items that vary the most and least widely across countries, by this measure, are shown in Table 5 . The items varying the most appear to be more negative than the items that varied the least. Among the most varying items are "People are disagreeing about something" and "Situation might make $\mathrm{P}$ feel inadequate"; among the least varying items are "Affords an opportunity for $\mathrm{P}$ to do things that might make $\mathrm{P}$ liked or accepted" and "Situation is potentially enjoyable." However, not all items in this table are so clearly positive or negative, and exceptions are visible as well; e.g., the least varying items include "Situation may cause feelings of hostility." In order to test this informally, imperfectly observed pattern, 18 of our non-U.S. lead research collaborators rated the 89 RSQ items on the dimension of positivity-negativity, ${ }^{6}$ using a 9-point scale with 1 representing "a negative experience" and 9 representing "a positive experience," and then we computed the average of all of the ratings (overall $\alpha=.98)$.

A simple $t$-test indicates that the 15 most varying items are indeed more negative than the 15 least varying items $(t$ 


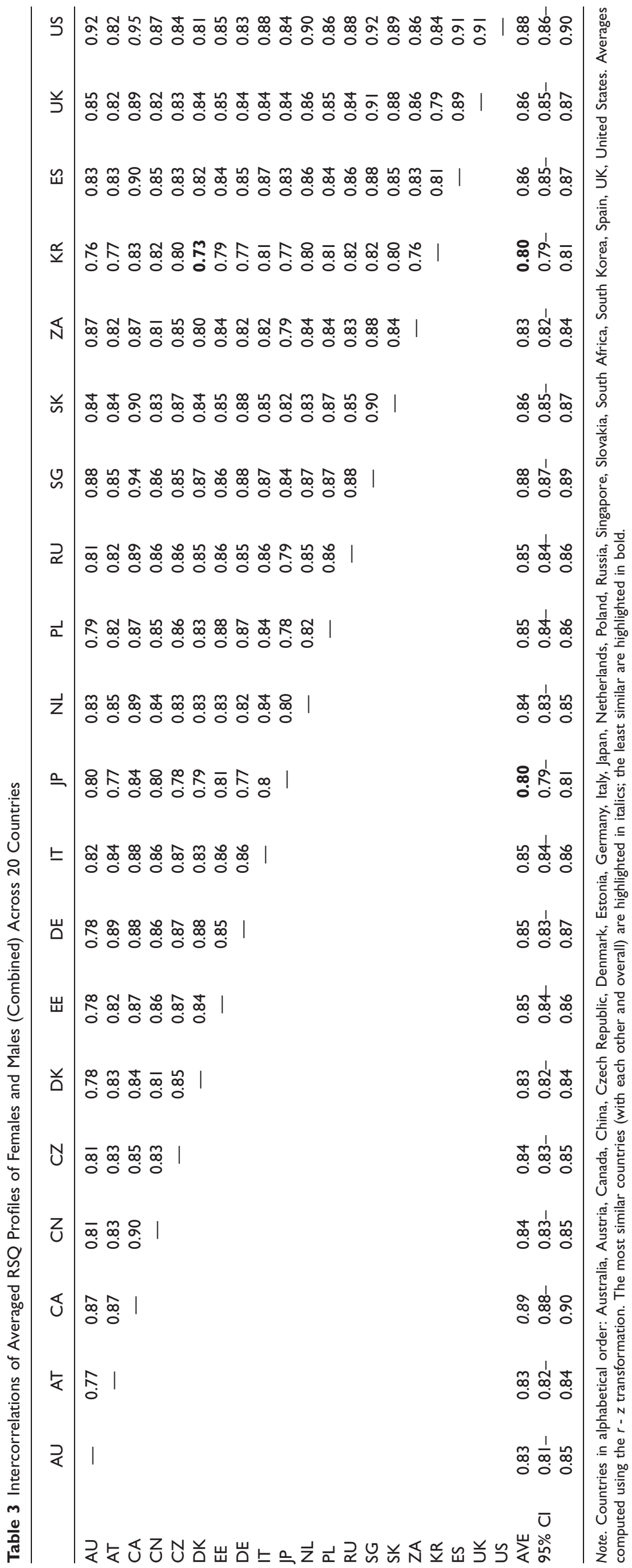


Table 4 Average Inter-Individual RSQ Correlations Within and Across 20 Countries

\begin{tabular}{|c|c|c|c|c|c|c|c|c|c|c|c|c|c|c|c|c|c|c|c|c|}
\hline & $\mathrm{AU}$ & AT & $C A$ & $\mathrm{CN}$ & $C Z$ & DK & $\mathrm{EE}$ & DE & IT & JP & NL & PL & RU & SG & SK & ZA & $\mathrm{KR}$ & ES & UK & US \\
\hline $\mathrm{AU}$ & 0.17 & 0.16 & 0.16 & 0.15 & 0.17 & 0.15 & 0.15 & 0.16 & 0.15 & 0.19 & 0.17 & 0.18 & 0.18 & 0.18 & 0.19 & 0.16 & 0.12 & 0.19 & 0.18 & 0.17 \\
\hline AT & & 0.18 & 0.18 & 0.17 & 0.19 & 0.17 & 0.17 & 0.20 & 0.17 & 0.19 & 0.19 & 0.20 & 0.19 & 0.18 & 0.20 & 0.17 & 0.14 & 0.20 & 0.18 & 0.16 \\
\hline CA & & & 0.18 & 0.17 & 0.18 & 0.16 & 0.17 & 0.18 & 0.16 & 0.19 & 0.18 & 0.20 & 0.19 & 0.19 & 0.19 & 0.18 & 0.13 & 0.20 & 0.18 & 0.17 \\
\hline $\mathrm{CN}$ & & & & 0.18 & 0.18 & 0.16 & 0.17 & 0.18 & 0.16 & 0.18 & 0.17 & 0.19 & 0.19 & 0.17 & 0.18 & 0.15 & 0.13 & 0.11 & 0.17 & 0.16 \\
\hline$C Z$ & & & & & 0.23 & 0.18 & 0.19 & 0.21 & 0.18 & 0.20 & 0.19 & 0.21 & 0.21 & 0.19 & 0.22 & 0.18 & 0.14 & 0.21 & 0.19 & 0.17 \\
\hline DK & & & & & & 0.18 & 0.17 & 0.19 & 0.16 & 0.19 & 0.17 & 0.19 & 0.19 & 0.17 & 0.18 & 0.16 & 0.12 & 0.19 & 0.18 & 0.15 \\
\hline $\mathrm{EE}$ & & & & & & & 0.19 & 0.19 & 0.16 & 0.19 & 0.18 & 0.20 & 0.19 & 0.18 & 0.19 & 0.16 & 0.13 & 0.19 & 0.18 & 0.16 \\
\hline $\mathrm{DE}$ & & & & & & & & 0.21 & 0.18 & 0.20 & 0.19 & 0.22 & 0.21 & 0.20 & 0.20 & 0.18 & 0.14 & 0.21 & 0.20 & 0.17 \\
\hline IT & & & & & & & & & 0.17 & 0.18 & 0.17 & 0.18 & 0.19 & 0.17 & 0.18 & 0.15 & 0.13 & 0.19 & 0.17 & 0.15 \\
\hline$J P$ & & & & & & & & & & 0.28 & 0.20 & 0.21 & 0.21 & 0.21 & 0.21 & 0.19 & 0.15 & 0.22 & 0.21 & 0.19 \\
\hline NL & & & & & & & & & & & 0.21 & 0.20 & 0.20 & 0.18 & 0.20 & 0.18 & 0.14 & 0.21 & 0.19 & 0.18 \\
\hline PL & & & & & & & & & & & & 0.24 & 0.22 & 0.20 & 0.22 & 0.19 & 0.16 & 0.22 & 0.21 & 0.19 \\
\hline RU & & & & & & & & & & & & & 0.23 & 0.20 & 0.22 & 0.19 & 0.16 & 0.22 & 0.20 & 0.19 \\
\hline SG & & & & & & & & & & & & & & 0.20 & 0.20 & 0.18 & 0.14 & 0.20 & 0.20 & 0.18 \\
\hline SK & & & & & & & & & & & & & & & 0.22 & 0.19 & 0.14 & 0.21 & 0.21 & 0.18 \\
\hline ZA & & & & & & & & & & & & & & & & 0.17 & 0.13 & 0.19 & 0.19 & 0.16 \\
\hline$K R$ & & & & & & & & & & & & & & & & & 0.12 & 0.15 & 0.14 & 0.13 \\
\hline ES & & & & & & & & & & & & & & & & & & 0.25 & 0.21 & 0.19 \\
\hline UK & & & & & & & & & & & & & & & & & & & 0.21 & 0.18 \\
\hline US & & & & & & & & & & & & & & & & & & & & 0.17 \\
\hline
\end{tabular}

Note. Countries in alphabetical order: Australia, Austria, Canada, China, Czech Republic, Denmark, Estonia, Germany, Italy, Japan, Netherlands, Poland, Russia, Singapore, Slovakia, South Africa, South Korea, Spain, UK, US. Figures on the diagonal in boldface represent within-country homogeneity.

$(28)=2.71, p=.011, r=-.46)$. It should be noted that this relationship applies primarily to the 15 most and least varying items; across all 89 items the correlation between positivity and the eta measure of cross-cultural variation is $r=-.15$, which does not (quite) attain conventional significance $(p=.08,95 \%$ CI $[-.35$, $06], d f=87)$. Nonetheless, it does appear that - in general and with some exceptions - the items varying the most across countries describe more negative situational experiences than the items that vary the least.

\section{Country-Level Variables and Attributes of Situational Experience}

The final step in data analysis was an exploratory investigation of the relationships among aspects of situational experience and country-level values, personality, economic output, and population. Previous research provided average scores for each of the 20 countries in our sample for six value dimensions: Power Distance, Individualism, Uncertainty Avoidance, Masculinity (also called Quantity of Life vs. Quality of Life and other labels), Long-term Orientation, and Indulgence (Hofstede, 1983; Hofstede Centre, n.d.). Average national scores on the Big Five personality traits were available for 16 of our 20 countries (Schmitt, Allik, McCrae, \& Benet-Martínez, 2007). For all 20 countries, we also obtained measures of Gross Domestic Product (United Nations, 2015), per-capita Gross Domestic Product (International Monetary Fund, 2014), and population (Central Intelligence Agency, 2014).

In exploratory research such as this, it is important to assess the number of significant correlations that would be expected by chance. For this purpose, we used the test described by Sherman and Funder (2009; see also Sherman \& Serfass, in press), in which the chance distribution of significant correlates is estimated over 10,000 randomized trials. This procedure allows estimation of the $p$-level of a list of correlates, taken as a set. Average value and personality scores were correlated with each of the 89 average RSQ placements for each country using this procedure.

Overall, only one value dimension and two Big Five personality traits generated more RSQ correlates than expected by chance. ${ }^{7}$ Individualism yielded 9 situational correlates $(p=.06)$, while the Big Five traits of Openness to Experience and Neuroticism both yielded 11 ( $p=.05$ and $p=.06$, respectively). These correlates appear in Tables 6, 7, and 8 .

We also assessed the correlation between RSQ elements of situational experience and two economic indicators, the national Gross Domestic Product (GDP) and the Gross Domestic Product per-Capita (GDPPC), as well as each country's population. Only the first of these variables generated more significant correlates than expected by chance (for GDP, the number of correlates expected by chance was 4.66 , and the number obtained was $11, p=.07$; for GDPPC, the number of significant correlates was 2 , fewer than expected by chance, $p=.85$; for population, the number of significant correlates was $8, p=.22$ ). The correlates of GDP appear in Table $9 .{ }^{8}$

Among other correlates, situations in countries with higher GDP were more likely to include someone being blamed, someone being dominated, and uncertainty; they were less likely to include behavioral limits or to include stimuli that could be construed sexually. Countries with high absolute GDP (as opposed to per-capita GDP) also tend to have higher populations (across the 20 countries in our sample, $r=.59, p<.01$ ). Even though the number of correlates of population did not exceed chance, each of the same correlations just listed for GDP are also 


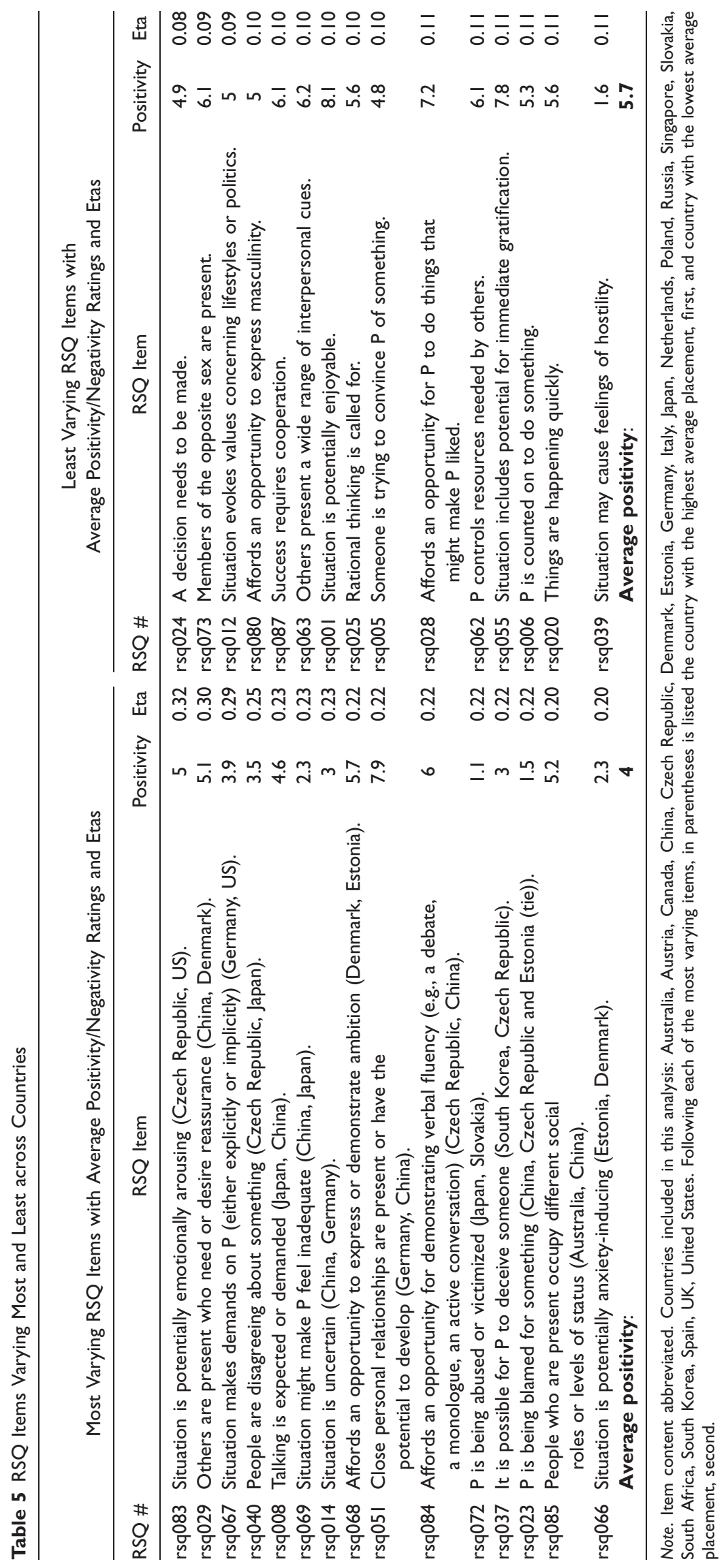


Table 6 Situational Correlates of Individualism Across 20 Countries

\begin{tabular}{|c|c|c|c|}
\hline Item \# & RSQ Item & $r$ & $p$-value \\
\hline rsq079 & Situation raises issues of power. & 0.80 & $* * *$ \\
\hline rsq034 & Situation includes one or more small annoyances. & 0.62 & $* *$ \\
\hline rsq016 & $\mathrm{P}$ is being criticized, directly or indirectly. & 0.53 & * \\
\hline rsq030 & Situation entails frustration. & 0.50 & * \\
\hline rsq060 & Situation is relevant to bodily health of $P$. & 0.47 & $*$ \\
\hline rsq04I & Affords an opportunity to express unusual ideas or points of view. & -0.73 & $* * *$ \\
\hline rsq083 & Situation is potentially emotionally arousing. & -0.69 & $* *$ \\
\hline rsq019 & Introspection is possible. & -0.50 & $*$ \\
\hline rsq025 & Rational thinking is called for. & -0.48 & $*$ \\
\hline
\end{tabular}

Note. $* * * * x<.001, * *=p<.01, *=p<.05$. The number of correlates $p<.05$ was 9 and the number expected by chance was $4.39(p=.06)$. Countries included in this analysis: Australia, Austria, Canada, China, Czech Republic, Denmark, Estonia, Germany, Italy, Japan, Netherlands, Poland, Russia, Singapore, Slovakia, South Africa, South Korea, Spain, UK, United States.

Table 7 Situational Correlates of Openness Across 16 Countries

\begin{tabular}{|c|c|c|c|}
\hline Item \# & RSQ Item & $r$ & $p$-value \\
\hline rsq08I & Others may need or are requesting advice from $\mathrm{P}$. & 0.75 & $* * *$ \\
\hline rsq040 & People are disagreeing about something. & 0.73 & $* *$ \\
\hline rsq038 & Someone else in this situation (other than P) might be deceitful. & 0.60 & $*$ \\
\hline rsq044 & Situation raises moral or ethical issues. & 0.58 & $*$ \\
\hline rsq033 & Situation would make some people tense and upset. & 0.58 & $*$ \\
\hline rsq070 & Situation includes stimuli that could be construed sexually. & 0.58 & * \\
\hline rsq056 & Social interaction is possible. & 0.55 & $*$ \\
\hline rsq029 & Others are present who need or desire reassurance. & -0.77 & $* * *$ \\
\hline rsq082 & Independence or autonomy of $\mathrm{P}$ is questioned or threatened. & -0.76 & $* * *$ \\
\hline rsq072 & $P$ is being abused or victimized. & -0.69 & $* *$ \\
\hline rsq008 & Talking is expected or demanded. & -0.52 & * \\
\hline
\end{tabular}

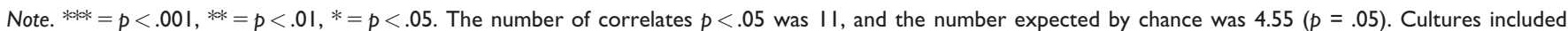
in this analysis: Australia, Austria, Canada, Czech Republic, Estonia, Germany, Italy, Japan, Netherlands, Poland, Slovakia, South Africa, South Korea, Spain, UK, United States.

significant, in the same direction, for population - and both sets of correlates are heavily influenced by the United States and China, which are relatively high in both population and GDP.

\section{DISCUSSION}

\section{Conclusions}

First, the situational experience of individuals around the world at 7:00 p.m. was, on average, highly similar and largely pleasant, and the homogeneity of individual situational experience was nearly as large between as within countries. This finding emerged even though the study examined situational experience in 20 countries, on 5 continents, using materials rendered in 14 different languages. While this degree of similarity may or may not be surprising, it is an encouraging indication that instructions to participants and the content of the RSQ items were indeed understood similarly across many different countries and languages.

Second, despite this overall similarity, specific aspects of situational experience did vary. All 89 of the RSQ items differed "significantly" across countries according to conven- tional analysis of variance-which is unsurprising given the large N. More interesting was an unpredicted finding that needs replication in future research: The items that varied the most across countries described more negative aspects of situations than the items varying the least. One reason may be that content and enforcement of social norms varies across cultural environments (Gelfand et al., 2011; Reno, Cialdini \& Kallgren, 1993). Thus, the experience of the negative situations such norms regulate may vary as well. In other words, perhaps Tolstoy was right; ${ }^{9}$ there may be more ways to be unhappy than to be happy — or, at least, negative aspects of situational experience appear more likely to vary across cultures than positive ones.

Finally, exploratory analyses examined the country-level correlations among situational experience and six dimensions of values and the Big Five personality traits, along with economic output and population. Although the $\mathrm{N}$ for these analyses - the number of countries in our sample for which these national scores were available - was small (16 for the traits and 20 for the other variables), one value dimension, two Big Five traits, and Gross Domestic Product correlated with more RSQ aspects of situational experience than expected by chance, according to 
Table 8 Situational Correlates of Neuroticism Across 16 Countries

\begin{tabular}{|c|c|c|c|}
\hline Item \# & RSQ Item & $r$ & $p$-value \\
\hline rsq029 & Others are present who need or desire reassurance. & $0.7 \mathrm{I}$ & ** \\
\hline rsq072 & $P$ is being abused or victimized. & 0.59 & $*$ \\
\hline rsq008 & Talking is expected or demanded. & 0.58 & $*$ \\
\hline rsq084 & Affords an opportunity for demonstrating verbal fluency. & 0.55 & $*$ \\
\hline rsq05I & Close personal relationships are present or have the potential to develop. & 0.50 & $*$ \\
\hline rsq070 & Situation includes stimuli that could be construed sexually. & -0.61 & $*$ \\
\hline rsq056 & Social interaction is possible. & -0.59 & * \\
\hline rsq069 & Situation might make $\mathrm{P}$ feel inadequate. & -0.55 & $*$ \\
\hline rsq040 & People are disagreeing about something. & -0.53 & * \\
\hline rsq033 & Situation would make some people tense and upset. & -0.52 & * \\
\hline rsq050 & Situation has potential to arouse guilt in $\mathrm{P}$. & -0.51 & $*$ \\
\hline
\end{tabular}

Note. $* *=p<.01, *=p<.05$. The number of correlates $p<.05$ was II, and the number expected by chance was $4.54(p=.06)$. Cultures included in this analysis: Australia, Austria, Canada, Czech Republic, Estonia, Germany, Italy, Japan, Netherlands, Poland, Slovakia, South Africa, South Korea, Spain, UK, United States.

Table 9 Situational Correlates of Gross National Product Across 20 Countries

\begin{tabular}{|c|c|c|c|}
\hline Item \# & RSQ Item & $r$ & $p$-value \\
\hline rsq023 & $P$ is being blamed for something. & 0.71 & $* * *$ \\
\hline rsq0I7 & Someone is attempting to dominate or boss $\mathrm{P}$. & 0.56 & $* *$ \\
\hline rsq014 & Situation is uncertain. & 0.53 & $*$ \\
\hline rsq054 & Assertiveness is required to accomplish a goal. & 0.47 & * \\
\hline rsq027 & Situation involves competition. & 0.47 & $*$ \\
\hline rsq080 & Affords an opportunity to express masculinity. & -0.80 & $* * *$ \\
\hline rsq064 & Situation includes behavioral limits. & -0.63 & $* *$ \\
\hline rsq073 & Members of the opposite sex are present. & -0.55 & $*$ \\
\hline rsq089 & Affords an opportunity to express femininity. & -0.54 & $*$ \\
\hline rsq085 & People who are present occupy different social roles or levels of status. & -0.53 & * \\
\hline rsq070 & Situation includes stimuli that could be construed sexually. & -0.52 & $*$ \\
\hline
\end{tabular}

Note. $* * *=p<.001, * *=p<.01, *=p<.05$. The number of correlates $p<.05$ was II, and the number expected by chance was 4.66 ( $p=.07$ ). Cultures included in this analysis: Australia, Austria, Canada, China, Czech Republic, Denmark, Estonia, Germany, Italy, Japan, Netherlands, Poland, Russia, Singapore, Slovakia, South Africa, South Korea, Spain, UK, United States.

randomization analyses (Sherman \& Funder, 2009; Sherman \& Sefass, in press).

The value dimension of Individualism is typically interpreted to reflect values such as "everybody is supposed to take care of him/herself and his/her immediate family," an "emphasis on individual initiative and achievement," and the idea that "everybody has a right to a private life and opinion" (Hofstede, 1983, p. 62). The positive correlates of this dimension include "Situation raises issues of power" and "P is being criticized," which seem compatible with the traditional definition. However, other, negative correlates include "Rational thinking is called for" and "Affords an opportunity to express unusual ideas or points of view." This last correlate (item 41), in particular, appears to go in the opposite direction to what the usual interpretation of Individualism would have led one to expect, and suggests that this cultural value and opportunities to express unusual views do not always go together. Closer examination of our data reveals that three countries, in particular, meet this description, largely accounting for the negative correlation. Denmark, Canada, and Australia are all relatively high in Individual- ism, but low on affording opportunities to express unusual ideas.

The country-level correlates of the Big Five trait of Openness to Experience are more theoretically sensible. Situations in countries high on this dimension are relatively likely to include people "disagreeing about something" (item 40), which could reflect lively intellectual exchange of the sort that might be expected in a culture where people, on average, are higher in Openness. Such exchanges might also include an element of deceit, advice giving, ethical issues, or even sexual topics. The situations in cultures where people are high on Openness are less likely to include abuse or victimization, questioning of autonomy, or including people who require reassurance. One country, Japan, was lowest both in average Openness and lowest on situations being characterized by disagreement; it is a culture that values consensus and works to avoid conflict. By contrast, the Czech Republic was relatively high on both items; its culture is characterized by energetic and sometimes intense debate. ${ }^{10}$

Most of the country-level correlates of Neuroticism also make theoretical sense. Situations in countries with higher 
Neuroticism scores (Japan is highest) were relatively likely to include people who need reassurance and feel abused; they are less likely to include sexual stimuli or for social interaction - a possible indication of loneliness. However, not all correlates fit this pattern. Situations in countries higher in Neuroticism also scored lower on making people feel inadequate, tense, or upset.

Further analyses explored national economic and population variables. While GDP was correlated with more situational aspects than would be expected by chance, per-capita GDP was not; nor was population. However, the correlates of population-relatively few as they were-overlapped with those of GDP, suggesting that larger countries in both economic output and population include more situations characterized by people being blamed and dominated, by uncertainty, and by a lack of behavioral limits. In particular, two countries with large GDP and large populations were characterized by high average placements on these RSQ items: the United States and China. Although these countries are on opposite sides of the "EastWest" divide often emphasized in cultural psychology, they do have in common a highly active and competitive economic environment.

\section{Limitations}

No prior study has comprehensively assessed situational experience around the world using a standardized assessment instrument. It should not be surprising, therefore, that this initial effort has several limitations.

First, and perhaps most obviously, the research uses a new instrument, the RSQ, for the assessment of situational experience. While using a common instrument across many contexts has the advantage of allowing comparisons across different areas of research, the inevitable trade-off is that it may not be ideal for any particular application. Moreover, researchers in the United States developed the original RSQ. Despite our care in translation through consultation with international colleagues, the measure could still be considered an "imposed etic" (Berry, 1980). Cross-cultural assessment of situations using dimensions developed by researchers from diverse countries would be a welcome addition to the literature. More generally, to the extent that interested researchers find important aspects of cultural variation missing from the RSQ, we would urge them to develop their own instruments to investigate how the findings of the present study are confirmed, contradicted, or extended when a wider measurement net is cast.

A second limitation is that all of the participants in this study were members of college communities, and for the most part students. This aspect of the data could be considered advantageous to the degree that it holds relatively constant many factors that might otherwise vary widely across cross-cultural samples, including age, socioeconomic status, and education level. This fact could give the present study a conservative bias, in that it might tend to underestimate cross-cultural differences to the extent that there is a global college "culture" (Flere \& Lavrič,
2008). It seems possible - but remains to be shown empirically - that broader samples of participants would yield larger differences in situational experience.

A third limitation is simply that all participants in this study described the situation they experienced at a particular time of day, 7:00 p.m. While we chose this time for a reason-it seemed to be an hour of the day in which activities were relatively free to vary - it is still just one moment. Ideally, we would have sampled each of our participants' situational experiences several times, on different days of the week, at different hours of the day (as was done in an earlier study using solely U.S. participants; Sherman et al., 2010). However, this did not seem feasible for an initial study being conducted in 20 different countries. For now, this study offers only a portrait of "the world at 7:00," a snapshot of situational experience at a particular time of day.

This brings us to a final limitation, which is that the study was based on assessments in "only" 20 countries. While that might seem like a good number - and one that we believe represents a decent start —other investigations of personality and culture have looked at even more (e.g., McCrae, 2002). In particular, our own sample, diverse as it is, lacks participants from Central and South America, India, and the Middle East. In addition, important cultural variation often can be found within nations (e.g., Allik et al., 2009; Tsai \& Chentsova-Dutton, 2003).

\section{Future Directions}

Building on this initial effort, the most obvious directions for future research entail overcoming the limitations just noted. New and different instruments for situational assessment should be applied, including ones specifically designed for cross-cultural application. Wider ranges of participants should be recruited, ideally nationally representative samples within each country, and also including cultural subgroups within large and diverse nations such as China, India, Russia, and the United States. Further efforts (which are, in fact, ongoing) should be made to recruit participants from under-represented areas of the world. Each participant should be asked to report on several situations experienced on different days of the week at varying times of day.

\section{The Active Ingredients of Culture}

The central data in this study reflect how participants described their recent, specific experience of situations. This is a distinctive aspect of the present research, because the data reflect assessments of cultural environments offered by the individuals who actually experience them-not by researchers trying to interpret unfamiliar cultural contexts from the point of view of visitors. This fact is critical, because the psychological impact of situations is mediated by how they are experienced (Brown, 1991; Oyserman, in press). By providing unprecedented findings about the degree to which situational experience is similar and different around the world, and the national-level aspects of 
personality, values, and environment that are associated with these differences, the present study offers unique insights into the active ingredients of culture, and opens new questions for future research.

\section{Declaration of Conflicting Interests}

The authors declared no potential conflicts of interest with respect to the research, authorship, and/or publication of this article.

\section{Funding}

The author(s) disclosed receipt of the following financial support for the research, authorship, and/or publication of this article: Martina Hřebíčková and Sylvie Graf were supported by grants from the Czech Science Foundation (13-25656S) and the Institute of Psychology, Academy of Sciences of the Czech Republic (RVO:68081740). Anu Realo was funded by the Estonian Ministry of Education and Science (IUT2-13). The overall organization of the data collection, at UC Riverside, was supported in part by National Science Foundation Grant BCS-1052638, David C. Funder, Principal Investigator. Any opinions, findings, and conclusions or recommendations expressed in this material are those of the individual researchers and do not necessarily reflect the views of the National Science Foundation or any other entity.

\section{Notes}

1. Exact definitions of "culture" vary and the issues are complex (Valsiner, 2003), but most (not all) "cross-cultural" research entails comparisons across different nations, the approach we take in the present study.

2. See He, Bartram, Inceoglu \& van de Vijver, 2014, for a recent exception.

3. The $d f$ of 87 is based on the correlation stemming from 89 pairs of averaged item ratings; this estimate should be viewed with caution because of the probable lack of independence among Q-sort items, which lowers the number of degrees of freedom to an unknown degree (Sherman \& Funder, 2009).

4. The $d f$ of 19 is for the mean of the 20 correlations between each country's average and the others.

5. CI's are reported here to three decimals to facilitate comparison with the between-country average.

6. None of the non-U.S. collaborators had seen these data at the time they made their ratings.

7. For purposes of these exploratory analyses, we used a threshold of $p<.10$.

8. The significant correlates of all the value, personality, economic, and population variables, along with the results of the respective randomization analyses, are reported in the supplementary materials.

9. "Happy families are all alike; every unhappy family is unhappy in its own way," Anna Karenina.

10. These cultural observations are from our Japanese and Czech coauthors, respectively.

\section{References}

Allik, J., Mõttus, R., Realo, A., Pullmann, H., Trifonova, A., McCrae, R.R., \& 56 Members of the Russian Character and Personality Survey. (2009). Personality traits of Russians from the observer's perspective. European Journal of Personality, 23, 567-588.

Bem, D.J., \& Funder, D.C. (1978). Predicting more of the people more of the time: Assessing the personality of situations. Psychological Review, 85, 485-501.

Berry, J.W. (1980). Introduction to methodology. In H.C. Triandis \& J.W. Berry (Eds.), Handbook of cross-cultural psychology: Methodology (Vol. 2, pp. 1-28). Boston: Allyn \& Bacon.

Block, J. (1978). The Q-sort method in personality assessment and psychiatric research. Palo Alto, CA: Consulting Psychologists Press. (Original work published 1961).

Block, J., \& Block, J.H. (1981). Studying situational dimensions: A grand perspective and some limited empiricism. In D.M. Magnusson (Ed.), Toward a psychology of situations: An interactional perspective (pp. 85-103). Hillsdale, NJ: Lawrence Erlbaum Associates.

Brown, D.E. (1991). Human universals. New York: McGraw-Hill.

Central Intelligence Agency. (2014). World factbook. Retrieved from https:/www.cia.gov/library/publications/the-world-factbook/ rankorder/2119rank.html

Ching, C.M., Church, T.A., Katigbak, M.S., Reyes, J.A.S., TanakaMatsumic, J., Takaokac, S., et al. (2013). The manifestation of traits in everyday behavior and affect: A five-country study. Journal of Research in Personality, 48, 1-16.

Church, A.T. (2010). Current perspectives in the study of personality across countries. Perspectives in Psychological Science, 5, 441449. doi: 10.1177/1745691610375559

Church, A.T., Katigbak, M. S., Ching, C.M., Zhang, H., Shen, J., Mazuera Arias, R., et al. (2013). Within-individual variability in self-concepts and personality states: Applying density distribution and situation/behavior approaches across countries. Journal of Research in Personality, 47, 922-935.

Church, A.T., Katigbak, M.S., \& del Prado, A.M. (2010). Cultural similarities and differences in perceived affordances of situations for Big Five behaviors. Journal of Research in Personality, 44, 78-90.

Edwards, J.A., \& Templeton, A. (2005). The structure of perceived qualities of situations. European Journal of Social Psychology, 35, 705-723.

Flere, S., \& Lavrič, M. (2008). On the validity of cross-cultural social studies using student samples. Field Methods, 20, 399-412.

Forgas, J.P., \& van Heck, G.L. (1992). The psychology of situations. In G. Caprara \& G.L. van Heck (Eds.), Modern personality psychology: Critical reviews and new directions (pp. 418-455). New York: Harvester-Wheatsheaf.

Frake, C.O. (1980). Language and cultural description: Essays by Charles O. Frake. Stanford, CA: Stanford University Press.

Funder, D.C. (2006). Towards a resolution of the personality triad: Persons, situations, and behaviors. Journal of Research in Personality, 40, 21-34. 
Funder, D.C. (2009). Persons, behaviors and situations: An agenda for personality psychology in the postwar era. Journal of Research in Personality, 43, 120-126.

Funder, D.C., Furr, R.M., \& Colvin, C.R. (2000). The Riverside Behavioral Q-sort: A tool for the description of social behavior. Journal of Personality, 68, 451-489.

Funder, D.C., \& Guillaume E. (2013). Revised RSQ for international research (version 3.15). Unpublished manuscript, University of California, Riverside.

Funder, D.C., Guillaume, E., Kumagi, S., Kawamoto, S., \& Sato, T. (2012). The person-situation debate and the assessment of situations. Japanese Journal of Personality, 21, 1-11.

Furr, R.M., Wagerman, S.A., \& Funder, D.C. (2010). Personality as manifest in behavior: Direct behavioral observation using the revised Riverside Behavioral Q-sort (RBQ-3.0). In C.R. Agnew, D.E. Carlston, W.G. Graziano, \& J.R. Kelly (Eds.), Then a miracle occurs: Focusing on behavior in social psychological theory and research (pp. 186-204). New York: Oxford University Press.

Gelfand, M.J. (2007, March 7). Situated culture: A multilevel analysis of situational constraint across 35 nations. Colloquium delivered at the University of California, Berkeley.

Gelfand, M.J., Raver, J.L., Nishii, L., Leslie, L.M., Lun, J., Lim, B.C., et al. (2011). Differences between tight and loose countries: A 33-nation study. Science, doi:10.1126/science.1197754

Gurven, M., von Rueden, C., Massenkoff, M., \& Kaplan, H. (2013). How universal is the Big Five? Testing the five-factor model of personality variation among forager-farmers in the Bolivian Andes. Journal of Personality and Social Psychology, 104, 354370 .

Haig, B.D. (2005). An abductive theory of scientific method. Psychological Methods, 10, 371-388.

Haig, B.D. (2014). Investigating the psychological world: Scientific method in the behavioral sciences. Cambridge, MA: MIT Press.

Heine, S.J., Lehman, D.R., Peng, K., \& Greenholtz, J. (2002). What's wrong with cross-cultural comparisons of subjective Likert scales? The reference-group effect. Journal of Personality and Social Psychology, 82, 903-918.

Hofstede Centre. (n.d.). Countries. Retrieved from http://geert-hofstede.com/countries.html

Hofstede, G. (1983). National cultures in four dimensions: A research-based theory of cultural differences among nations. International Studies of Management and Organizations, 13, 46-74.

Hřebíčková, M., \& Graf, S. (2014). Accuracy of national stereotypes in central Europe: Outgroups are not better than ingroups in considering personality traits of real people. European Journal of Personality, 28, 60-72. doi:10.1002/per.1904

International Monetary Fund. (2014). World Economic Outlook Data Base. Retrieved from http://www.imf.org/external/pubs/ft/weo/ 2014/02/weodata/index.aspx

Kelley, H.H., Holmes, J.G., Kerr, N.L., Reis, H.T., Rusbult, C.E., \& van Lange, P.A.M. (2003). An atlas of interpersonal situations. New York: Cambridge University Press.

Kitayama, S., Markus, H.R., Matsumonot, H., \& Narasakkunkit, V. (1997). Individual and collective processes in the construction of the self: Self-enhancement in the United States and self-criticism in Japan. Journal of Personality and Social Psychology, 72, 1245-1267.

Kitayama, S., Mesquita, B., \& Karasawa, M. (2006). Cultural affordances and emotional experience: Socially engaging and disengaging emotions in Japan and the United States. Journal of Personality and Social Psychology, 91, 890-903.

Kluckhohn, C., \& Murray, H.A. (1953). Personality in nature, society, and country. New York: Alfred A. Knopf.

Magnusson, D. (1971). An analysis of situational dimensions. Perceptual and Motor Skills, 32, 851-867.

Markus, H.R., \& Kitayama, S. (1998). The cultural psychology of personality. Journal of Cross-cultural Psychology, 29, 63-87.

Masuda, T., \& Nisbett, R.E. (2001). Attending holistically versus analytically: Comparing the context sensitivity of Japanese and Americans. Journal of Personality and Social Psychology, 81, 922-934.

McCrae, R.R. (2002). NEO-PI-R data from 36 cultures. In R R. McCrae \& J. Allik (Eds.), The five factor model of personality across cultures (pp. 105-126). New York: Plenum Publishers.

McCrae, R.R., \& Allik, J. (Eds). (2002). The Five-Factor Model of personality across countries. New York: Kluwer Academic/Plenum Publishers.

McCrae, R.R., Chan, W., Jussim, L., De Fruyt, F., Löckenhoff, C.E., De Bolle, M., et al. (2013). The inaccuracy of national character stereotypes. Journal of Research in Personality, 47, 831-842.

McCrae, R.R., \& Costa, P.T. (1997). Personality trait structure as a human universal. American Psychologist, 52, 509-516.

Morse, P., Neel, R., Todd, E., \& Funder, D.C. (2014). Renovating situation taxonomies: Exploring the construction and content of fundamental motive situation types. Journal of Personality, doi: 10.1111/jopy. 12111

Oyserman, D. (in press). Culture as situated cognition. Emerging Trends in the Social Sciences.

Realo, A., Allik, J., Lonnqvist, J.E., Verkasalo, M., Kwiatkowska, A., Kööts, L., et al. (2009). Mechanisms of the national character stereotype: How people in six neighboring countries of Russia describe themselves and the typical Russian. European Journal of Personality, 23, 229-249.

Reis, H.T. (2008). Reinvigorating the concept of situation in social psychology. Personality and Social Psychology Review, 12, 311329.

Reno, R.R., Cialdini, R.B., \& Kallgren, C.A. (1993). The transsituational influence of social norms. Journal of Personality and Social Psychology, 64, 104-112. doi:10.1037/0022-3514.64. 1.104

Ross, C.E., \& Mirowsky, J. (1984). Socially-desirable response and acquiescence in a cross-cultural survey of mental health. Journal of Health and Social Behavior, 25, 189-197.

Saucier, G., Bel-Bahar, T., \& Fernandez, C. (2007). What modifies the expression of personality tendencies? Defining basic domains of situational variables. Journal of Personality, 75, 479-504.

Schmitt, D.P. Allik, J., McCrae, R.R., \& Benet-Martínez, V. (2007). The geographic distribution of Big Five personality traits: 
Patterns and profiles of human self-description across 56 nations. Journal of Cross-cultural Psychology, 38, 173-212.

Serfass, D.G., \& Sherman, R.A. (2013). Personality and perceptions of situations from the Thematic Apperception Test. Journal of Research in Personality, 47, 708-718.

Sherman, R.A., \& Funder, D.C. (2009). Evaluating correlations in studies of personality and behavior: Beyond the number of significant findings to be expected by chance. Journal of Research in Personality, 99, 330-343.

Sherman, R.A., Nave, C.N., \& Funder, D.C. (2010). Situational similarity and personality predict behavioral consistency. Journal of Personality and Social Psychology, 99, 330-343.

Sherman, R.A., Nave, C.S., \& Funder, D.C. (2012). Properties of persons and situations related to overall and distinctive personality-behavior congruence. Journal of Research in Personality, 46, 87-101.

Sherman, R.A., Nave, C.S., \& Funder, D.C. (2013). Situational construal is related to personality and gender. Journal of Research in Personality, 47, 1-14.

Sherman, R.A., \& Serfass, D.G. (in press). The comprehensive approach to analyzing multivariate constructs. Journal of Research in Personality.

Shweder, R.A. (1991). Thinking through countries: Expeditions in cultural psychology. Cambridge, MA: Harvard University Press.

ten Berge, M.A., \& De Raad, B. (1999). Taxonomies of situations from a trait psychological perspective: A review. European Journal of Personality, 13, 337-360.

Terracciano, A., Abdel-Khalek, A.M., Ádám, N., Adamovová, L., Ahn, C.K., Ahn, H.-N., et al. (2005). National character does not reflect mean personality trait levels in 49 countries. Science, $\mathbf{3 1 0}$, 96-100.

Todd, E., \& Funder, D.C. (2012, January). Personality correlates of unique perceptions of brief film situations. Paper presented at the Annual Meetings of the Society for Personality and Social Psychology, San Diego.

Triandis, H.C. (1996). The psychological measurement of cultural syndromes. American Psychologist, 51, 407- 415. doi:10.1037/ 0003-066X.51.4.407

Tsai, J., \& Chentsova-Dutton, Y. (2003). Variation among European Americans in emotional facial expression. Journal of Crosscultural Psychology, 34, 650-657.

United Nations Statistics Division. (2015). GDP and its breakdown at current prices in US Dollars. New York: United Nations.

Valsiner, J. (2003). Culture and its transfer: Ways of creating general knowledge through the study of cultural particulars. In W.J. Lonner, D.L. Dinnel, S.A. Hayes, \& D.N. Sattler (Eds.), Online readings in psychology and culture (Unit 2, Chapter 12). Bellingham, WA: Center for Cross-Cultural Research, Western Washington University. Retrieved from http://www.wwu.edu/culture/ Valsiner.htm

Van Heck, G.L. (1984). The construction of a general taxonomy of situations. In H. Bonarius, G.L. Van Heck, \& N. Smid (Eds.), Personality psychology in Europe: Theoretical and empirical developments (pp. 149-164). Lisse: Swets and Zeitlinger. van de Vijver, F.J.R., \& Leung, K. (1997). Methods and data analysis for cross-cultural research. Newbury Park, CA: Sage.

van de Vijver, F.J.R., \& Leung, K. (2011). Equivalence and bias: A review of concepts, models, and data analytic procedures. In D. Matsumoto \& F.J.R. van de Vijver (Eds.), Cross-cultural research methods in psychology (pp. 17-45). New York: Cambridge University Press.

Wagerman, S.A., \& Funder, D.C. (2009). Personality psychology of situations. In P.J. Corr \& G. Matthews (Eds.), Cambridge handbook of personality (pp. 27-42). Cambridge: Cambridge University Press.

Yang, Y., Read, S.J., \& Miller, L.C. (2006). A taxonomy of situations from Chinese idioms. Journal of Research in Personality, 40, 750-778.

\section{APPENDIX}

Author affiliations are listed below:

Esther Guillaume, University of California, Riverside Erica Baranski, University of California, Riverside Elysia Todd, University of California, Riverside Brock Bastian, University of Queensland Igor Bronin, Ural Federal University Christina Ivanova, Ural Federal University Joey T. Cheng, University of California, Berkeley François S. de Kock, University of Cape Town Jaap J.A. Denissen, Tilburg University David Gallardo-Pujol, University of Barcelona Peter Halama, Institute of Psychology, Slovak Academy of Sciences

Gyuseog Q. Han, Chonnam National University Jaechang Bae, Chonnam National University Jungsoon Moon, Chonnam National University Ryan Y. Hong, National University of Singapore Martina Hřebíčková, Academy of Sciences of the Czech Republic

Sylvie Graf, Academy of Sciences of the Czech Republic

Paweł Izdebski, Kazimierz Wielki University

Lars Lundmann, University of Copenhagen

Lars Penke, Georg August University, Göttingen

Marco Perugini, University of Milan

Giulio Costantini, University of Milan

John Rauthmann, Humboldt University, Berlin

Matthias Ziegler, Humboldt University, Berlin

Anu Realo, University of Tartu

Liisalotte Elme, University of Tartu

Tatsuya Sato, Ritsumeikan University

Shizuka Kawamoto, Ritsumeikan University

Piotr Szarota, Institute of Psychology, Polish Academy of Sciences

Jessica L. Tracy, University of British Columbia

Marcel A.G. van Aken, University of Utrecht

Yu Yang, China Europe International Business School

David C. Funder, University of California, Riverside 


\section{SUPPORTING INFORMATION}

Additional Supporting Information may be found in the online version of this article at the publisher's web-site, or at www.rap.ucr.edu/pub17.pdf.

Significant RSQ Correlates of Power Distance, across countries

Significant RSQ Correlates of Masculinity, across countries

Significant RSQ Correlates of Long-term Orientation, across countries
Significant RSQ Correlates of Extraversion, across countries

Significant RSQ Correlates of Agreeableness, across countries

Significant RSQ Correlates of Openness, across countries Significant RSQ Correlates of per-capita GNP, across countries

Mean RSQ Placement of 15 most varying items, for each of 20 countries

Mean RSQ Placement of 15 least varying items, for each of 20 countries 